\title{
Method of the Year 2011
}

The ability to introduce targeted, tailored changes into the genomes of several species will make it feasible to ask more precise biological questions.

In our annual toast to biological research methods and to the scientists who develop them, we have chosen genome editing with engineered nucleases as our Method of the Year 2011.

Perhaps the most reliable way to learn about the function of a gene or protein is to specifically perturb it and monitor what happens. This reverse genetic approach is routinely applied in many species, but, with a few exceptions, it is challenging or even impossible to make targeted changes at endogenous genomic loci, arguably the most elegant method of genetic perturbation. Instead, the experimenter must settle for more indirect approaches: overexpressing the modified gene from a heterologous location or knocking it down, often only partially, with an approach such as RNA interference. Engineered nucleases-which can be designed, in principle, to cut at any location in the genome of any species and thus to introduce tailored modifications into the endogenous sequence-are set to change this. We provide a brief Primer on these tools on page 27 .

From the perspective of methods development, the trajectory of the engineered nucleases has been a compelling one, as reported in a News Feature on page 23. All three major classes of these enzymes-zinc-finger nucleases (ZFNs), transcription activator-like effector nucleases (TALENs) and engineered meganucleases-stand on the shoulders of very basic studies, a testament, yet again, to the necessity of basic research and the difficulty in predicting whence technological leaps will come. Engineering the first ZFN to modify an endogenous gene in an organism (the fruit fly, as it appositely turns out), required that three strands of knowledge, gained from many decades of research in many laboratories, be drawn together: how restriction endonucleases work, how DNA breaks are repaired by the cell and how DNA-binding proteins achieve specificity in the vastness of genomic sequence space.

This said, it has undoubtedly been the potential clinical utility of these tools - to correct mutations in monogenic human disease, for instance-that has primarily driven the intense effort put into developing them over the past decades. The fortuitous consequence, however, is that very powerful basic research tools have been generated in the process.

Engineered nucleases can be used to knock out or knock in genes, to make allelic mutants, to change gene-regulatory control and to add reporters or epitope tags, all in the endogenous genomic context. Matthew Porteus discusses these and other exciting research applications of these tools in a Commentary on page 28.

Although the first ZFN was reported more than a decade ago, the pace of work in this field has picked up remarkably in the past year. This is in no small part because of the development of TALENs. ZFNs remain the best-characterized tools, but they are not always easy to design, as Mark Isalan discusses in a Commentary on page 32 . The recent discovery of TAL effectors in plant pathogenic bacteria and the realization that their properties-notably their apparently simpler DNA-binding code-should mitigate some of the existing problems with engineering robust tools, has given a real boost to the field. Gene-editing nucleases will achieve their full potential when they can be easily and quickly designed, in practice, to specifically modify any sequence of any genome; having more than one technology available will help achieve this goal.

In addition, commercially available ZFNs are dropping in price at the same time as methods developers are assembling tools to help researchers design their own nucleases. Meanwhile, TALENs and engineered meganucleases are also already commercially available. Which technology will dominate is not yet clear: there are still many unknowns, in particular about TALEN function as discussed in several of the pieces in this issue. But the price at which researchers in regular research labs can obtain good tools is likely to play a role.

You can also hear about genome editing with engineered nucleases in a short video, and we include in this issue, as in previous years, a section of Methods to Watch in the future (p. 35).

To all our readers, a happy, successful 2012! 